\title{
Optimizing functional accuracy of TMS in cognitive studies: a comparison of methods
}

Citation for published version (APA):

Sack, A. T., Kadosh, K. C., Schuhmann, T., Moerel, M., Walsh, V., \& Goebel, R. (2009). Optimizing functional accuracy of TMS in cognitive studies: a comparison of methods. Journal of Cognitive Neuroscience, 21(2), 207-221. https://doi.org/10.1162/jocn.2009.21126

Document status and date:

Published: 01/01/2009

DOI:

10.1162/jocn.2009.21126

Document Version:

Publisher's PDF, also known as Version of record

Document license:

Taverne

Please check the document version of this publication:

- A submitted manuscript is the version of the article upon submission and before peer-review. There can be important differences between the submitted version and the official published version of record.

People interested in the research are advised to contact the author for the final version of the publication, or visit the DOI to the publisher's website.

- The final author version and the galley proof are versions of the publication after peer review.

- The final published version features the final layout of the paper including the volume, issue and page numbers.

Link to publication

\footnotetext{
General rights rights.

- You may freely distribute the URL identifying the publication in the public portal. please follow below link for the End User Agreement:

www.umlib.nl/taverne-license

Take down policy

If you believe that this document breaches copyright please contact us at:

repository@maastrichtuniversity.nl

providing details and we will investigate your claim.
}

Copyright and moral rights for the publications made accessible in the public portal are retained by the authors and/or other copyright owners and it is a condition of accessing publications that users recognise and abide by the legal requirements associated with these

- Users may download and print one copy of any publication from the public portal for the purpose of private study or research.

- You may not further distribute the material or use it for any profit-making activity or commercial gain

If the publication is distributed under the terms of Article $25 \mathrm{fa}$ of the Dutch Copyright Act, indicated by the "Taverne" license above, 


\title{
Optimizing Functional Accuracy of TMS in Cognitive Studies: A Comparison of Methods
}

\author{
Alexander T. Sack ${ }^{1}$, Roi Cohen Kadosh², Teresa Schuhmann", \\ Michelle Moerel ${ }^{1}$, Vincent Walsh ${ }^{2}$, and Rainer Goebel ${ }^{1}$
}

\begin{abstract}
Transcranial magnetic stimulation (TMS) is a tool for inducing transient disruptions of neural activity noninvasively in conscious human volunteers. In recent years, the investigative domain of TMS has expanded and now encompasses causal structure-function relationships across the whole gamut of cognitive functions and associated cortical brain regions. Consequently, the importance of how to determine the target stimulation site has increased and a number of alternative methods have emerged. Comparison across studies is precluded because different studies necessarily use different tasks, sites, TMS conditions, and have different goals. Here, therefore, we systematically compare four commonly used TMS coil positioning approaches by using them to induce behavioral change in a single cognitive study. Specifically, we investigated the behavioral impact of right parietal TMS during a number comparison task, while basing TMS localization either on (i) individual fMRI-guided TMS neuronavigation, (ii) individual MRI-guided TMS neuronavigation, (iii) group functional
\end{abstract}

\section{INTRODUCTION}

Transcranial magnetic stimulation (TMS) allows controlled manipulation of brain activity, with a quantifiable impact on behavior or cognition (Sack, 2006; Hallett, 2000; Pascual-Leone, Walsh, \& Rothwell, 2000; PascualLeone, Bartres-Faz, \& Keenan, 1999), and is now a wellestablished tool for inducing transient disruptions of neural activity noninvasively in conscious human volunteers (Di Lazzaro et al., 2004; Robertson, Theoret, \& Pascual-Leone, 2003; Walsh \& Pascual-Leone, 2003; Walsh \& Cowey, 2000). In recent years, TMS has expanded its investigative domain, which has brought to the forefront questions of how to optimally target stimulation sites in individual subjects, and to establish optimal and sufficient methods. Some areas, when stimulated, have a signature output: TMS over the motor cortex causes a twitch (Ziemann, 2004), and TMS over the visual cortex can induce phosphenes (Stewart, Battelli, Walsh, \& Cowey, 1999; Epstein, Verson, \& Zangaladze, 1996; Epstein \&

\footnotetext{
${ }^{1}$ Maastricht University, Maastricht, The Netherlands, ${ }^{2}$ University College London, London, UK
}

Talairach coordinates, or (iv) 10-20 EEG position P4. We quantified the exact behavioral effects induced by TMS using each approach, calculated the standardized experimental effect sizes, and conducted a statistical power analysis in order to calculate the optimal sample size required to reveal statistical significance. Our findings revealed a systematic difference between the four approaches, with the individual fMRI-guided TMS neuronavigation yielding the strongest and the P4 stimulation approach yielding the smallest behavioral effect size. Accordingly, power analyses revealed that although in the fMRI-guided neuronavigation approach five participants were sufficient to reveal a significant behavioral effect, the number of necessary participants increased to $n=9$ when employing MRI-guided neuronavigation, to $n=13$ in case of TMS based on group Talairach coordinates, and to $n=47$ when applying TMS over P4. We discuss these graded effect size differences in light of the revealed interindividual variances in the actual target stimulation site within and between approaches.
Zangaladze, 1996). However, many of the areas of interest in cognitive TMS studies, for example, the posterior parietal cortex, the occipital face area, the extrastriate body area, the prefrontal cortex, the inferior frontal gyrus, and so forth, are behaviorally silent (Penfield, 1958) and do not produce an immediately observable response. Thus, localization of target stimulation sites and positioning of the TMS coil presents a problem.

One common approach of localizing the TMS target stimulation site of "silent" brain areas is to place the coil according to the International 10-20 electrode scalp positioning system (Jasper, 1958). Although this method may be sufficient for some purposes, it does not take into account interindividual differences in the functional architecture of the brain or differences in the correspondence between scalp landmarks and underlying brain anatomy (Okamoto et al., 2004; Herwig, Satrapi, \& Schonfeldt-Lecuona, 2003). With the introduction of frameless stereotaxic systems, the TMS coil could be navigated to target specific anatomical sites based on individual subjects' structural brain images. This requires a TMS neuronavigation system, which creates a virtual link between MR images and real anatomy, and allows 
three-dimensional (3-D) orientation by interactive visual navigation (Herwig et al., 2002; Gugino et al., 2001; Boroojerdi et al., 1999; Paus, 1999; Ettinger et al., 1998; Krings et al., 1997). However, although this approach accounts for interindividual differences in anatomical brain structures, it does not account for individual differences in structure-function relationships. Stereotaxic neuronavigation can also be based on fMRI coordinates reported in the literature (probabilistic approach; Paus et al., 1997). Hence, a third approach of positioning the TMS coil is to use frameless stereotaxy in order to navigate the TMS coil to specific standardized brain coordinates, for instance, Talairach coordinates that have been reported in the literature to be activated during tasks similar to those to be used in a TMS experiment. The coordinates used are therefore generated either from group functional imaging data, or based on a meta-analysis of several studies using different imaging techniques. This approach considers both structural and functional imaging data relevant to the cognitive task to be used, but is still prey to individual structure-function differences. Finally, stereotaxic neuronavigation may be based on a subject's individual fMRI data for the respective cognitive function. Based on these individual imaging results, TMS subsequently probes whether the identified task-correlated activities in these areas are necessary for successful task performance (Andoh et al., 2006; Sack, Kohler, Linden, Goebel, \& Muckli, 2006; Thiel et al., 2005). This fourth approach thus accounts for interindividual differences both in brain anatomy and in the functional architecture of the brain.

The question to be answered is how much pre-TMS anatomy is sufficient to make inferences based on TMS. This gives rise to the question of how much effort is required to optimize the accuracy of TMS coil positioning given the limits of spatial resolution of TMS. Sparing, Buelte, Meister, Paus, and Fink (2008) have addressed this question by evaluating the accuracy and efficiency of different localization strategies for TMS-based primary motor cortex mappings (Sparing et al., 2008) and found the highest precision with fMRI-guided stimulation, which was accurate within the range of millimeters. These findings thus indicate that even in case of simple TMS motor mapping measurements, the strength and the accuracy of the induced effects are systematically modulated by the way the TMS coil is positioned above the target site. The importance of coil positioning becomes more problematic when applying TMS to silent areas in studies of cognitive functions. A recent study by Feredoes, Tononi, and Postle (2007) illustrated this point by using fMRI to localize TMS sites to disrupt short-term retention of verbal information. The authors demonstrated that fMRI-guided stereotaxic neuronavigation revealed significant effects only when based on the subject's individual fMRI data, whereas the determination of the TMS target site based on spatially normalized group-average fMRI data indicated a "wrong" brain region
(Feredoes et al., 2007). Feredoes et al. (2007) based their conclusion on a dichotomized all-or-nothing principle (i.e., fMRI-guided neuronavigation based on single-subject analyses reveals TMS effects, whereas fMRI-guided neuronavigation based on group analyses does not). However, it may be much more realistic to expect that the difference between various coil positioning approaches is gradual rather than qualitative. In order to address these issues, a systematic and quantitative assessment of the differential effect of different TMS coil positioning approaches on the induced behavioral changes during cognitive studies is needed.

In a recent cognitive TMS study (Cohen Kadosh et al., 2007), we demonstrated that a TMS-induced disruption of the right, but not left, intraparietal sulcus (IPS) significantly impairs automatic magnitude processing as measured by a reduced facilitatory component of the size congruity effect (SCE). During this study, we investigated a small number of participants $(n=5)$ with maximum precision and a high number of trials, emphasizing exact individual TMS coil positioning using fMRI-guided neuronavigation. We believed that the imaging-guided TMS neuronavigation was a methodological prerequisite for optimally revealing the specific and significant behavioral effects of right parietal TMS on magnitude processing. However, without a direct comparison between fMRI-guided TMS neuronavigation with other ways of targeting TMS stimulation sites, the importance of our methodological approach on the effect strength and sample size was speculative. It thus also remained unclear to what extent the revealed TMS-induced automatic number processing impairments during right parietal TMS may depend on how we determined target stimulation site. In the current study, we investigated whether the effects of right parietal TMS on size congruity revealed in our previous study can be obtained when using other approaches to determine the target stimulation site. We repeated our original study testing the same number of participants $(n=5)$ under four different experimental conditions, comparing the four methods of determining the target stimulation site. We hypothesized that the strengths of the behavioral effects of TMS in cognitive studies depend on the use of an appropriate localization strategy for positioning the TMS coil above the "silent" target stimulation site.

\section{METHODS}

\section{Participants}

Twenty healthy volunteers ( 7 men, mean age $=24$ years, $S D=3.9)$ were assigned to four experimental groups of five participants. A between-subject design had to be employed because preliminary psychophysical tests showed a strong learning effect (i.e., better task performance with practice). To avoid confounds between TMS effects and task familiarity, four separate groups of 
randomly assigned subjects were tested. Each group underwent one method of coil localization. All participants were medically approved, had normal or corrected-tonormal vision, and had no history of neurological or psychiatric disorders. All gave written informed consent after being introduced to the procedure. The study was approved by the local Medical Ethical Commission.

\section{Stimuli and Paradigm}

Stimuli were two digits (vertical visual angle of $0.8^{\circ}$ or $1.1^{\circ}$ ) presented centrally. The center-to-center distance between the two digits subtended $4^{\circ}$ horizontally. Presentation (Neurobehavioral Systems, San Francisco, CA) stimulus-presentation software was used. Both digits varied in numerical value as well as in physical size. Participants were instructed to decide which of two stimuli in a given display was numerically larger while ignoring the physical size difference between both numbers. The numerical comparisons were performed with congruent, neutral, or incongruent digit pairs. In the congruent condition, one digit is numerically and physically larger or smaller, (e.g., 2 4). In the incongruent condition, one digit is physically larger, whereas the other is numerically larger, or vice versa (e.g., 2 4). In the neutral condition, there is no difference in physical size (e.g., 2 4). Participants were instructed to indicate as quickly and accurately as possible which digit is numerically larger by pressing one of two keys corresponding to the side of the display. Previous studies show that participants unintentionally process the irrelevant dimension (Cohen Kadosh, Lammertyn, \& Izard, 2008; Cohen Kadosh et al., 2007; Tzelgov, Meyer, \& Henik, 1992; Henik \& Tzelgov, 1982), resulting in decreased response times to congruent trials (facilitation) and increased response times to incongruent trials (interference) when compared to the neutral condition.

\section{Magnetic Resonance Imaging}

Prior to TMS, high-resolution anatomical images were obtained for each participant using a 3-T magnetic resonance imaging scanner (Siemens Allegra MR Tomograph; Siemens AG, Erlangen, Germany). The T1-weighted dataset was acquired with the help of a magnetizationprepared rapid acquisition gradient-echo (MP-RAGE) sequence or a T1-weighted structural scan with an isotropic resolution of $1 \mathrm{~mm}$ using a modified driven equilibrium Fourier transform (MDEFT) sequence with optimized contrast for gray matter and white matter and imaging parameters $($ echo time $=4 \mathrm{msec}, 256 \times 256 \times 192$ matrix, voxel dimensions $=1 \times 1 \times 1 \mathrm{~mm}$ ).

\section{Cortical-surface Reconstruction}

MR data were analyzed using the BrainVoyager QX 1.8 software package (BrainInnovation, Maastricht, The
Netherlands). The high-resolution anatomical recordings were used for surface reconstruction of the right hemisphere of each participant. The surface reconstruction was performed in order to recover the exact spatial structure of the cortical sheet and to improve the visualization of the anatomical gyrification. The white-gray matter boundary was segmented with a region growing method preceded by inhomogeneity correction of signal intensity across space. The borders of the two resulting segmented subvolumes were tessellated to produce a surface reconstruction of the right hemisphere. Subsequently, inflated cortical surfaces were created.

\section{fMRI Measurements and Analysis}

In five participants, the MR measurements also included the acquisition of functional images while subjects performed the size congruity paradigm described above. The main purpose of these fMRI measurements was to localize the exact brain activity changes underlying the described SCE individually in each participant. Functional images were acquired using a gradient-echo echoplanar imaging sequence (16 axial slices; repetition time/ echo time $=2500 / 30 \mathrm{msec}$; flip angle $=90^{\circ}$; field of view $=192 \times 192 \mathrm{~mm}$; voxel size $=3 \times 3 \times 5 \mathrm{~mm})$. Each functional run contained 48 trials $(24$ trials $\times 2$ congruencies [incongruent/congruent]). Stimulus presentation was synchronized with the fMRI sequence at the beginning of each trial. The first two volumes of each run were discarded to allow for T1 equilibration. The remaining functional datasets were coregistered to Talairachtransformed anatomical data (Talairach \& Tournoux, 1988) and 3-D motion-corrected for each participant. The 3-D functional dataset was resampled to a voxel size of $3 \times 3 \times 3 \mathrm{~mm}$. Further preprocessing included spatial smoothing with a Gaussian kernel (full width at half maximum $[\mathrm{FWHM}]=8 \mathrm{~mm}$ for the group analysis and $\mathrm{FWHM}=3 \mathrm{~mm}$ for the individual analysis), linear trend removal, temporal high-pass filtering (high pass: $0.00647 \mathrm{~Hz}$ ), and autocorrelation removal. The predictor time courses (boxcar functions) were convolved with a gamma distribution to account for the shape and delay of the hemodynamic response (Boynton, Engel, Glover, \& Heeger, 1996). Each trial began with an asterisk fixation point, presented for $500 \mathrm{msec}$ at the center of the screen. Five hundred milliseconds after the fixation point disappeared, a pair of digits appeared for $1 \mathrm{sec}$ (Figure 1). The intertrial interval was $6000 \mathrm{msec}$, and the interblock interval was at least $15 \mathrm{sec}$. Stimuli were projected (Sanyo PLC-XT16) onto a frosted screen at the rear end of the scanner bore and viewed through a mirror mounted onto the head coil. Participants' responses were registered by a hand-held fiber-optic response system (LUMItouch fMRI Optical Response keypad, Photon Control, Burnaby, Canada; www.photonixco.com). At the group level, a fixed-effect analysis was employed, with a $2 \times 2$ factorial repeated measures design. Group effects 
Figure 1. Size congruity paradigm. Each pair of stimuli was preceded by a fixation point and a blank screen (500 msec each) and remained visible for $1 \mathrm{sec}$. After $6 \mathrm{sec}$ (intertrial interval), a new trial began with the presentation of a fixation point. The participants had to decide which stimulus was numerically larger while ignoring the physical size of the stimulus. The correct response was indicated by a button press.

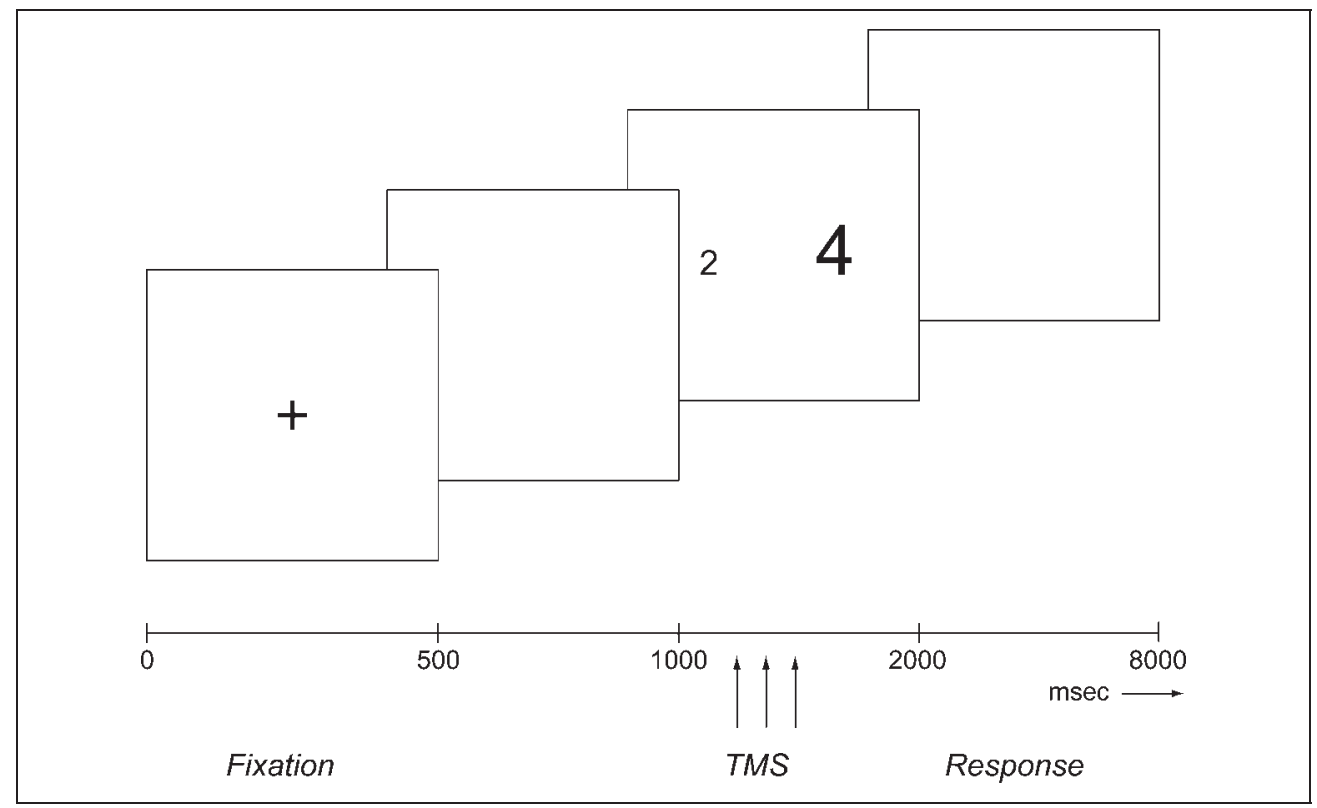

are shown if the $p$ value yielded $p<.05$ (Bonferronicorrected for multiple comparisons). For the individual data, the effects are shown if the $p$ value yielded $p<.001$ (uncorrected).

\section{Coregistration of Stereotaxic and MRI Data}

For TMS recording and positioning we used the BrainVoyager TMS Neuronavigation System (BrainInnovation, Maastricht, The Netherlands). This system consists of several miniature ultrasound transmitters attached to the participant's head and to the TMS coil. These ultrasound markers continuously transmit pulses to a receiving sensor device. Local spatial coordinate systems are created by linking the relative raw spatial position of the ultrasound senders to a set of fixed additional landmarks on the participant's head. The specification of these fixed landmarks is achieved via a digitizing pen that also hosts two transmitting ultrasound markers in order to measure its relative position in 3-D space. The three anatomical landmarks used to define the local coordinate system were the nasion and the two incisurae intertragicae. The neuronavigation system thus provides topographic information of the head-based transmitters relative to a participant-based coordinate frame. After having defined the local spatial coordinate system for the participant's head and the TMS coil in real 3-D space, these coordinate systems have to be coregistered with the coordinate system of the MR space. For TMS-MRI coregistration, the same landmarks digitized on the participant's head are specified on the head reconstruction of the anatomical data from the MR sequence. After the landmarks specified on the real head have been coregistered with those on the reconstructed head, events occurring around the head of the participant in real space are registered on- line, and are visualized and recorded in real-time at correct positions relative to the participant's anatomical reconstruction of the brain. The TMS coil can now be neuronavigated to a specific anatomical brain area or functional region of interest in every individual participant (Figure 2).

\section{Localization of TMS Target Region}

In accordance with previous findings using the identical size congruity paradigm (Cohen Kadosh et al., 2007,

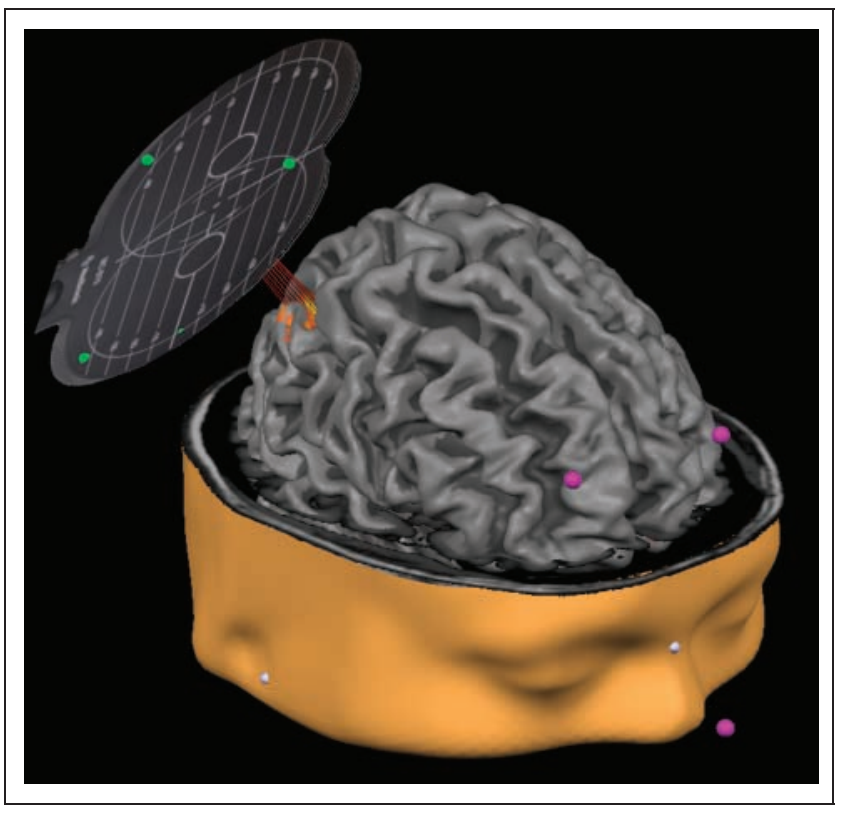

Figure 2. TMS neuronavigation based on individual fMRI data. An example of functional data superimposed on the anatomical reconstruction of the brain and the coregistered TMS coil. 
2008; Kaufmann et al., 2005; Pinel, Piazza, Le Bihan, \& Dehaene, 2004), we aimed to apply TMS to the right IPS in order to interfere with automatic magnitude processing. In our previous study, we demonstrated that a TMS-induced disruption of right, but not left, IPS activity significantly impairs the SCE as measured by a significant decrease in the response speed facilitation during congruent stimuli. In the current study, however, we wanted to systematically investigate whether this previously revealed TMS-induced behavioral effect following fMRIguided TMS neuronavigation over the right IPS could also be revealed with other methods of determining the right parietal target stimulation site. Therefore, we repeated our original study testing the same number of participants $(n=5)$ under four different experimental conditions, systematically comparing the four different most commonly used approaches of determining the parietal target stimulation site in TMS studies. We intentionally decided to test only five subjects in each of the approaches in order to investigate whether all approaches were able to reveal significant results when testing such a small number of subjects, as individual fMRI-based stereotaxic neuronavigation had accomplished.

\section{Four Different Approaches of Determining the Parietal TMS Site}

\section{fMRI-guided TMS Neuronavigation}

For one group, the right parietal TMS target site was determined as the individual location within the IPS exhibiting the strongest BOLD-signal contrast for the SCE. In order to achieve this precision, the functional data of each individual participant were superimposed on the individual anatomical reconstruction of the brain. In every participant, the TMS coil was then neuronavigated using frameless stereotaxy in order to stimulate the specific functional activation area in the right IPS underlying the SCE. In contrast to the fMRI group analysis, TMS neuronavigation was based on individual data in AC-PC space (the cerebrum was rotated into the anterior commissure-posterior commissure plane). This was done in order to avoid any additional transformations that could distort the correspondence between MRI and stereotaxic points.

\section{MRI-guided TMS Neuronavigation}

The parietal TMS target site for the second group was determined based on individual MRI data. For each participant, an anatomical reconstruction of the right hemisphere was created. Prior to the experiment, the target site was individually determined as the anterior part of the IPS. The TMS neuronavigation system was employed to navigate to this anatomically defined target region in each participant.
TMS Neuronavigation Based on Group

\section{Talairach Coordinates}

For the third group, the parietal TMS target site was determined as the Talairach coordinates of the location in the fMRI group analysis which exhibited the strongest SCE within the right IPS (Cohen Kadosh et al., 2007; Talairach coordinates $[x, y, z]=[22,-68,39])$. These normalized stereotaxic coordinates were marked as target sites on the normalized brain of each participant. This location was then back-transformed to the individual subject's brain coordinates in native space by reversing the native-to-Talairach transformation procedure. Finally, the TMS neuronavigation system was used to navigate to this group-average target site on the basis of each individual brain anatomy in AC-PC space.

\section{TMS Based on the 10-20 EEG System (Anatomical Landmark Approach)}

For the fourth group, the International 10-20 System for EEG electrode placement (Jasper, 1958) was employed for determination of the parietal TMS target site. A lycra swimming cap was placed on the participant's head in order to mark the location of P4, which has been shown to correspond to the right IPS (Herwig et al., 2003). Although placement of the TMS coil required no TMS neuronavigation, it was still used in order to determine in every single participant which exact anatomical brain structure was actually underlying $\mathrm{P} 4$, and thus, stimulated during this approach.

\section{Experimenter Bias}

The stereotaxic system employed in this study allows real-time and on-line TMS neuronavigation to any predefined TMS target site. Importantly, the accuracy of coil positioning is monitored and recorded throughout the entire experiment. Therefore, it is certain that a target site is stimulated equally precise in all four conditions, excluding the possibility that differential results between conditions are due to differences in stimulation accuracy, for example, due to random fluctuations or an experimenter bias.

\section{TMS Protocol}

Biphasic TMS pulses were applied with a figure-of-eight coil (MCB70; inner and outer radii of the two coil loops = 1.2 and $5.4 \mathrm{~cm}$, respectively) and a Medtronic MagPro X100 stimulator (Medtronic Functional Diagnostics A/S, Skovlunde, Denmark; maximum stimulator output $=$ $2 \mathrm{~T})$. The coil was fixed on a tripod and placed tangentially on the skull with a custom-made coil holder. The coil position was monitored on-line using the BrainVoyager TMS Neuronavigation System. Triple-pulse TMS at $10 \mathrm{~Hz}$ was applied at $60 \%$ of maximum stimulator 
output. The pulses were given at 220, 320, and $420 \mathrm{msec}$ after stimulus presentation. The timing of the pulses was chosen based on a recent ERP study that found increased amplitudes in the congruency conditions at the parietal electrode around $220 \mathrm{msec}$ after stimulus presentation (Walsh \& Cowey, 2000). These amplitudes peaked around 350-400 msec after stimulus presentation. For the sham condition, TMS pulses were applied using a placebo figure-of-eight TMS coil (MC-P-B70 Placebo). The positioning and navigation of the placebo coil, as well as the applied stimulation parameters, were the same as those in the real TMS condition. Each participant underwent a TMS session, in which the order of real and sham stimulation was counterbalanced between the participants. Congruent, neutral, and incongruent conditions were randomly sampled, and there was an equal sampling for each condition. A total of 144 trials were presented to each participant $(24$ trials $\times 2$ sessions [TMS, sham] $\times 3$ congruencies [incongruent, neutral, and congruent]). Responses had to be made equally often with the left and right hand.

\section{Statistical Analysis of Behavioral Data}

Mean RTs for every participant in each condition were calculated for correct trials only. RTs that were 2.5 standard deviations from the mean of each condition for each individual were excluded (less than 2\%). An outlier analysis was performed per approach (group mean \pm $2.5 S D)$, ensuring that the group means were not distorted by extreme values of a single subject. RTs were analyzed for all four approaches together, and subsequently, for the conditions separately using a $2 \times 3$ factorial repeated measures ANOVA (Stimulation [TMS/ sham] $\times$ Congruency [incongruent/neutral/congruent]) . In order to assess the facilitatory effect more directly, a $2 \times 2$ factorial repeated measures ANOVA was performed (Stimulation [TMS/sham] $\times$ Congruency [neutral/ congruent]). Additionally, one-sample $t$ tests were performed on the difference between sham and TMS in SCE (calculated as RT [incongruent] - RT [congruent]) for each of the approaches separately, which allowed an estimation of the effect size obtained by each of the four approaches.

\section{RESULTS}

\section{Behavioral Results without TMS: The Size Congruity Effect}

The task performance during baseline elicited a strong and significant SCE as measured by a significant difference between congruent and incongruent trials both in terms of RTs [RT (incongruent) - RT (congruent) = $104 \mathrm{msec}, S D=44.3 ; t=10.530, p$ (one-tailed) $<.001]$ and error rates [error rate (incongruent) - error rate (congruent $)=0.200, S D=0.142 ; t=6.320, p$ (one-tailed $)<$
.001]. This significant SCE was composed of a significant facilitatory component [RT (neutral) - RT (congruent) $=$ $36 \mathrm{msec}, S D=20.9 ; t=7.766, p$ (one-tailed) $<.001]$ as well as a significant interference component [RT (incongruent) - RT (neutral) $=68 \mathrm{msec}, S D=41.4 ; t=7.346$, $p$ (one-tailed $)<.001]$. Whereas the facilitatory effect reveals that participants were significantly faster in their decision when the task-irrelevant dimension of the stimuli (physical size) was in accordance with the taskrelevant dimension (numerical size), the interference effect demonstrates a significant decrease in performance when the task-irrelevant dimension was in conflict with the task-relevant dimension.

\section{fMRI Data}

fMRI was employed in order to localize the exact neural correlates underlying this behavioral SCE. A contrast analysis between congruent and incongruent trials during fMRI revealed higher activation in bilateral premotor regions (the putative frontal eye fields and the presupplementary motor area) and the right frontal operculum. Several of these areas have been reported in studies that utilized conflict situations (Critchley, Tang, Glaser, Butterworth, \& Dolan, 2005; Aron, Robbins, \& Poldrack, 2004; Rushworth, Walton, Kennerley, \& Bannerman, 2004; Picard \& Strick, 2001). Moreover, in line with previous studies (Kaufmann et al., 2005; Pinel et al., 2004), the SCE activated the IPS bilaterally (Figure 3). Our previous study ascertained that within this bilateral parietal activation network only the right IPS activity is functionally necessary for the facilitatory component of the SCE as revealed by fMRI-guided TMS neuronavigation (Cohen Kadosh et al., 2007). Based on this result, we targeted only the right IPS in the subsequent TMS studies, aiming to replicate our previous findings of a TMS-induced reduction of the facilitatory component of the SCE to compare the four different approaches of targeting the exact stimulation site within the right IPS: (i) fMRI-guided TMS neuronavigation, (ii) MRI-guided TMS neuronavigation, (iii) TMS neuronavigation based on group Talairach coordinates, and (iv) TMS over P4.

\section{Effects of fMRI-guided TMS Neuronavigation}

In order to accurately stimulate the functionally defined region of interest along the right IPS in each participant, the IPS SCE of the fMRI data was analyzed separately for each individual participant. The results revealed considerable interindividual differences in the IPS activation both in extent and in spatial layout and, therefore, in the stimulated coordinates (Figure 3; Table 2).

\section{Sham TMS}

When stimulating this functionally defined right IPS activation using a TMS placebo coil (sham condition), we 
Figure 3. Individual differences in brain activity. The different colors represent the neural correlates underlying the behavioral SCE for the individual participants.

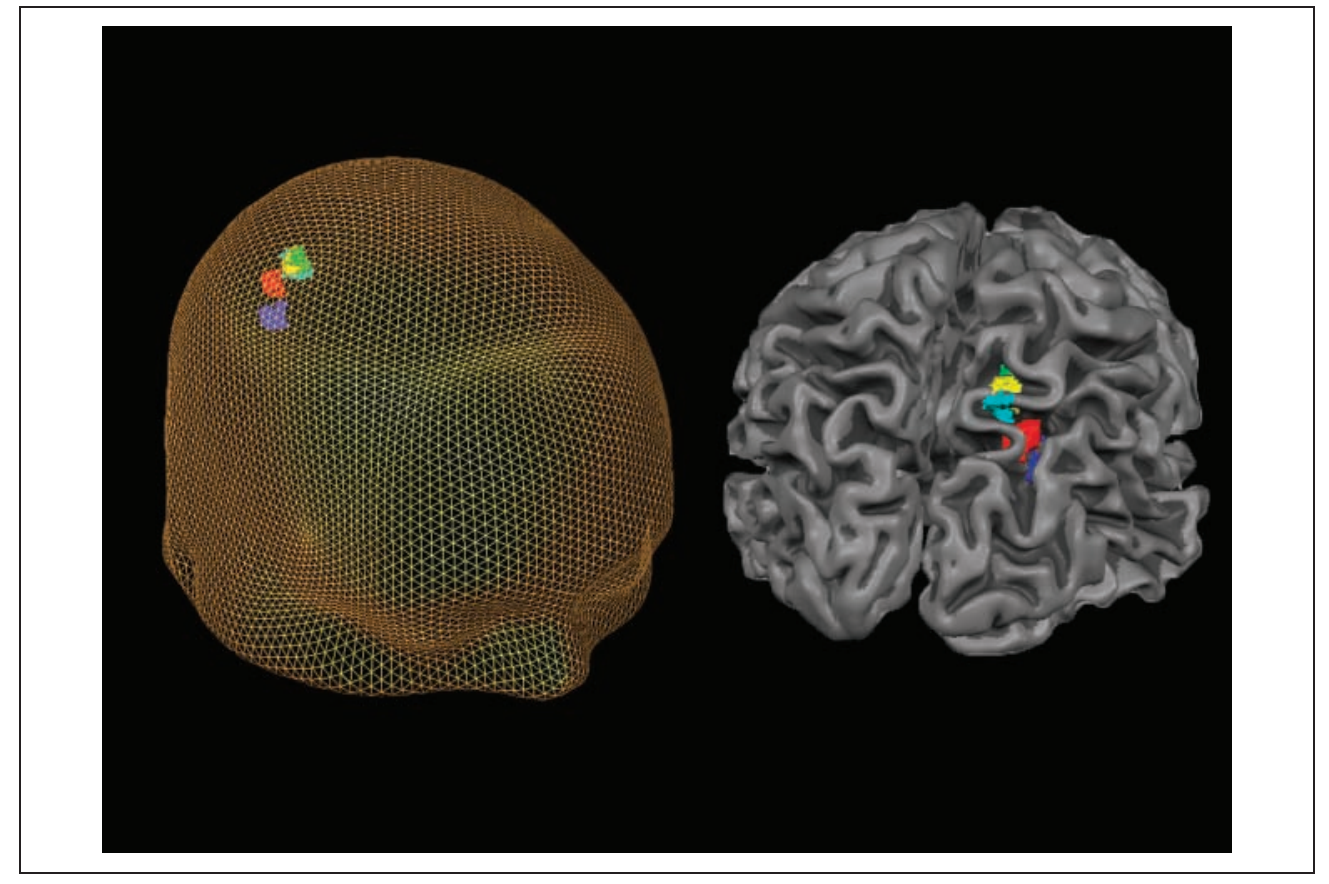

again revealed a clear and significant SCE $[t=4.073$, $p$ (one-tailed) $<.01$ ], calculated as [RT (incongruent) - RT (congruent)]. More concretely, RTs during congruent trials (466 msec, $S D=39.4$ ) were significantly faster as compared to incongruent trials $(579 \mathrm{msec}, S D=88.7)$. This SCE comprised both a significant facilitatory component [ $44 \mathrm{msec}, S D=4.6 ; t=21.465$, $p$ (one-tailed) $<$ .001], as well as a significant interference effect [69 msec, $S D=62.9 ; t=2.467, p($ one-tailed $)<.05]$.

\section{Right IPS}

A direct statistical contrast between fMRI-guided real versus sham TMS over the right IPS revealed that during real TMS, the overall SCE [RT (incongruent) - RT (congruent $)=80 \mathrm{msec}, S D=48.0]$ was significantly reduced $[t=2.517, p$ (one-tailed) $<.05$; see Figure 4] as compared to sham TMS [RT (incongruent) - RT (congruent) $=113 \mathrm{msec}, S D=62.4 \mathrm{]}$. Importantly, a two-way repeated measures ANOVA with TMS (sham vs. real TMS) and congruency (neutral or congruent) as the two within-subject factors revealed a significant interaction between TMS and congruency $[F(1,4)=7.677$, $p<.05]$.

\section{Effects of MRI-guided TMS Neuronavigation}

\section{Sham TMS}

During sham TMS, we again revealed a clear and significant SCE $[t=39.617, p$ (one-tailed) $<.001]$. More concretely, RTs during congruent trials (439 $\mathrm{msec}, S D=$ 37.0) were significantly faster as compared to incongruent trials $(564 \mathrm{msec}, S D=37.2)$. This SCE was composed of both, a significant facilitatory component [43 msec,
$S D=24.4 ; t=3.899, p($ one-tailed $)<.05]$, as well as a significant interference effect $[82 \mathrm{msec}, S D=20.9 ; t=$ 8.743, $p$ (one-tailed) $<.01]$.

\section{Right IPS}

When stimulating this anatomically defined right IPS using real TMS, we also revealed a significant SCE $[t=$ 8.143, $p$ (one-tailed) <.001]. Although the SCE was descriptively reduced during real TMS [RT (incongruent) $\mathrm{RT}$ (congruent) $=102 \mathrm{msec}, S D=27.9]$ as compared to sham TMS [RT (incongruent) - RT (congruent) = $124 \mathrm{msec}, S D=7.0$ ], a direct post hoc contrast between

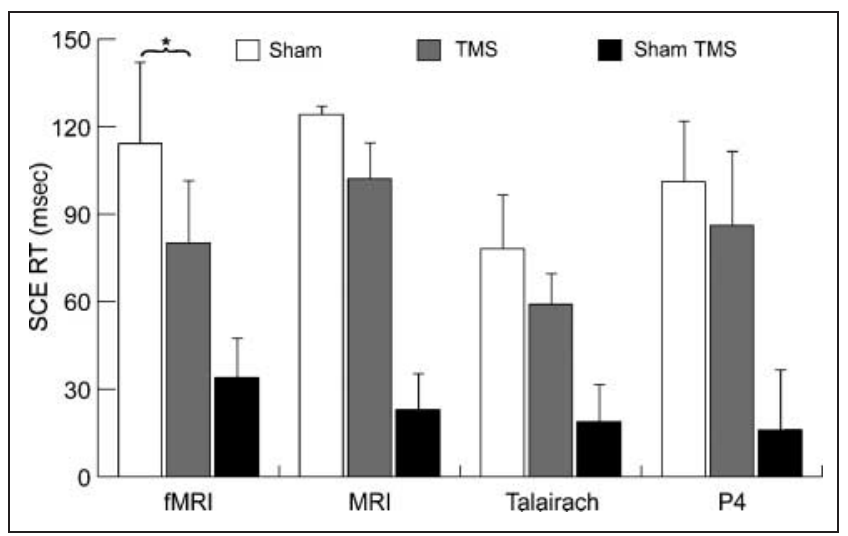

Figure 4. Size congruity effect as a function of the four approaches for TMS target site determination. White, gray, and black bars reflect the SCE for sham, TMS, and the difference between sham and TMS, respectively. Error bars depict one standard error of the mean $(S E M)$. The asterisk indicates a significant difference between SCE (sham) and SCE (TMS) at $\alpha=.05$. 
both TMS conditions did not find this difference to be significant $[t=1.834, p$ (one-tailed) $=.071$; see Figure 4]. Accordingly, a two-way repeated measures ANOVA with TMS (sham vs. real TMS) and congruency (neutral or congruent) as the two within-subject factors also failed to reveal a significant interaction $[F(1,4)=$ 2.504, $p=.189$ ].

\section{Effects of TMS Neuronavigation Based on Talairach Coordinates}

\section{Sham TMS}

During sham TMS, we revealed a clear and significant SCE $[t=4.183, p$ (one-tailed) $<.01]$. More concretely, RTs during congruent trials ( $407 \mathrm{msec}, S D=33.1$ ) were significantly faster as compared to incongruent trials ( $484 \mathrm{msec}, S D=71.3$ ). This SCE was composed of both a significant facilitatory component $[25 \mathrm{msec}, S D=24.3$; $t=2.284, p$ (one-tailed) $<.05$ ], as well as a significant interference effect [ $53 \mathrm{msec}, S D=36.6$; $t=3.235$, $p$ (onetailed) $<.05$ ].

\section{Right IPS}

When stimulating the right IPS based on Talairach group coordinates using real TMS, we also revealed a significant SCE $[t=5.548, p$ (one-tailed) $<.01]$. Although the SCE was descriptively reduced during real TMS [RT (incongruent) $-\mathrm{RT}$ (congruent) $=59 \mathrm{msec}, S D=23.7]$ as compared to sham TMS [RT (incongruent) - RT (congruent $)=78 \mathrm{msec}, S D=41.6]$, a direct post hoc contrast between both TMS conditions did not find this difference to be significant $[t=1.487, p$ (one-tailed $)=$ .106; see Figure 4]. Accordingly, a two-way repeated measures ANOVA with TMS (sham vs. real TMS) and congruency (neutral or congruent) as the two withinsubject factors also failed to reveal a significant interaction $[F(1,4)=0.100, p=.542]$.

\section{Effects of TMS over P4}

\section{Sham TMS}

During sham TMS, we revealed a clear and significant SCE $[t=4.891, p$ (one-tailed) $<.01]$. More concretely, RTs during congruent trials ( $426 \mathrm{msec}, S D=56.0$ ) were significantly faster as compared to incongruent trials (528 msec, $S D=90.5)$. This SCE was composed of both a significant facilitatory component $[34 \mathrm{msec}, S D=23.8$; $t=3.159, p$ (one-tailed) $<.05]$, as well as a significant interference effect [68 $\mathrm{msec}, S D=43.4 ; t=3.494$, $p$ (one-tailed $)<.05]$.

\section{Right IPS}

When stimulating P4 based on the 10-20 EEG system using real TMS, we also revealed a significant SCE $[t=$
3.338, $p$ (one-tailed) <.05]. Although the SCE was descriptively reduced during real TMS [RT (incongruent) $\mathrm{RT}$ (congruent) $=86 \mathrm{msec}, S D=57.5]$ as compared to sham TMS [RT (incongruent) - RT (congruent) = $101 \mathrm{msec}, S D=46.4$ ], a direct post hoc contrast between both TMS conditions did not find this difference to be significant $[t=0.749, p$ (one-tailed) $=.247$; see Figure 4]. Accordingly, a multivariate two-way repeated measures ANOVA with TMS (sham vs. real TMS) and congruency (neutral or congruent) as the two within-subject factors also failed to reveal a significant interaction $[F(1$, 4) $=0.833, p=.413]$.

\section{Directly Comparing the Effect Sizes of the Four Approaches}

Our results indicate that when the right IPS is stimulated in the location indicated by the individual fMRI results, the SCE decreases significantly. Importantly, this effect was only found to be significant with $n=5$ when using fMRI-guided TMS neuronavigation to approach the TMS target site, but could not be replicated using a different approach for TMS target site determination testing the same number of participants. Although all applied TMS approaches showed the same trend as the fMRI-guided TMS neuronavigation, the effect size was not large enough to reach statistical significance. In the next stage of analysis, we computed the power of these experiments to determine how many subjects would be needed for each of the three reductions in SCE to become significant.

In contrast to just comparing significance differences between groups, the effect size is a standardized measure of the real effect, which takes into account not only the observed difference in RTs but also the variability, the alpha error, the beta error, the test power, and the sample size, making it a valid measure of the standardized strength of any observed effect (Cohen, 1988). Importantly, effect sizes enable a parametric differentiation and ordering within and between several significant or nonsignificant results.

When directly comparing the four approaches for TMS target site determination, a clear difference occurs in the strength of the effect size of TMS (Table 1). As discussed in the previous section, for all four approaches

Table 1. Effect Sizes of Paired-samples $t$ Tests on the Difference in SCE between Sham and TMS

\begin{tabular}{ll}
\hline & Effect Size (Cohen's d) \\
\hline fMRI & 1.13 \\
MRI & 0.82 \\
Talairach & 0.67 \\
Anatomical & 0.34 \\
\hline
\end{tabular}


the SCE was reduced in the TMS condition as compared to the sham condition. However, when calculating the effect size of this difference, the effect in the fMRI-guided TMS neuronavigation was largest (Cohen's $d=1.13$ ), followed by the effect size for the MRI-guided TMS neuronavigation (Cohen's $d=0.82$ ), followed by the effect size for the TMS neuronavigation based on group Talairach coordinates (Cohen's $d=0.67$ ), and finally, smallest for the TMS over P4 approach (Cohen's $d=$ 0.34). The effect sizes for the interaction between TMS and congruency as shown by the two-way repeated measures ANOVA with TMS (sham or TMS) and congruency (incongruent, neutral, or congruent) as within-subject factors showed the same order in effect sizes between the four approaches. Again, fMRI-guided TMS neuronavigation generated the largest effect size $\left(f^{2}=1.23\right)$, followed by MRI-guided TMS neuronavigation $\left(f^{2}=\right.$ 0.91), neuronavigation based on Talairach coordinates $\left(f^{2}=0.34\right)$, and TMS stimulation over P4 $\left(f^{2}=0.13\right)$.

\section{Optimal Sample Size}

The optimal sample size of an experiment can theoretically be defined as the exact number of subjects necessary to reveal an a priori determined empirical effect of a certain size, or any greater size, as statistically significant. This implies that any smaller empirical effect, although potentially statistically significant at a greater sample size, will not reach statistical significance under these circumstances. Statistical power analysis enables the determination of an optimal sample size by a priori specifying the $\mathrm{H} 1$ parameter, and thus, controlling not only for the alpha $(\alpha)$ - but also the beta $(\beta)$-error probability of the statistical analysis. Speaking in statistical terms, the exact predetermination of an $\mathrm{H} 0$ and an $\mathrm{H} 1$ parameter enables one to calculate the probability of an empirical effect (and all higher effects) in case of a valid H0 ( $\alpha$-error probability), as well as to calculate the probability of an empirical effect (and all smaller effects) in case of a valid $\mathrm{H} 1$ ( $\beta$-error probability). The significance level $(\alpha)$, the test power $(1-\beta)$, the experimental effect $\left(\eta^{2}\right)$, and the sample size $(n)$ are interdependent, and thus, after determining any three of these parameters, it is possible to calculate the fourth. In the current study, the significance level for both $\alpha$ - and $\beta$-error probability are conventionally defined. Moreover, we calculated the exact standardized effect size for each of the four approaches. Hence, three of the four parameters are set, enabling the calculation of the exact number of participants required for yielding each of the described effect sizes as being significant. This power analysis revealed that while in the fMRI-guided neuronavigation experiment, five participants were sufficient for finding a significant difference between sham and TMS, this number of necessary participants increased to $n=9$ when employing MRI-guided neuronavigation, to $n=13$ in case of TMS based on group Talairach coordinates, and finally, to $n=47$ when applying TMS over P4 (Figure 5). Hence, although all four approaches reveal the same direction of effect, they differ in the number of participants needed to make any claims regarding statistical significance.

\section{Quantification of Individual Variability in TMS Target Site}

Although all four approaches of determining the optimal TMS target stimulation site attempted to denote the same area within the right IPS as target site, their systematic difference in TMS-induced behavioral effect sizes suggests that this has not been the case. In order to quantify the difference in the actual stimulation site between approaches that might give rise to these systematic effect size differences, the coordinates of the exact actual stimulation site in each individual participant were assessed and analyzed using frameless stereotaxy (Figure 6; Table 2). As can be seen in Table 2 (see also Figure 6), the actual TMS target site was considerably different in each participant. During the fMRI-guided
Figure 5. Relation between sample size and alpha $(\alpha)$ value for each of the four approaches. The different markers indicate the four approaches for determination of TMS stimulation site. The horizontal line indicates the alpha $(\alpha)$ value of .05 .

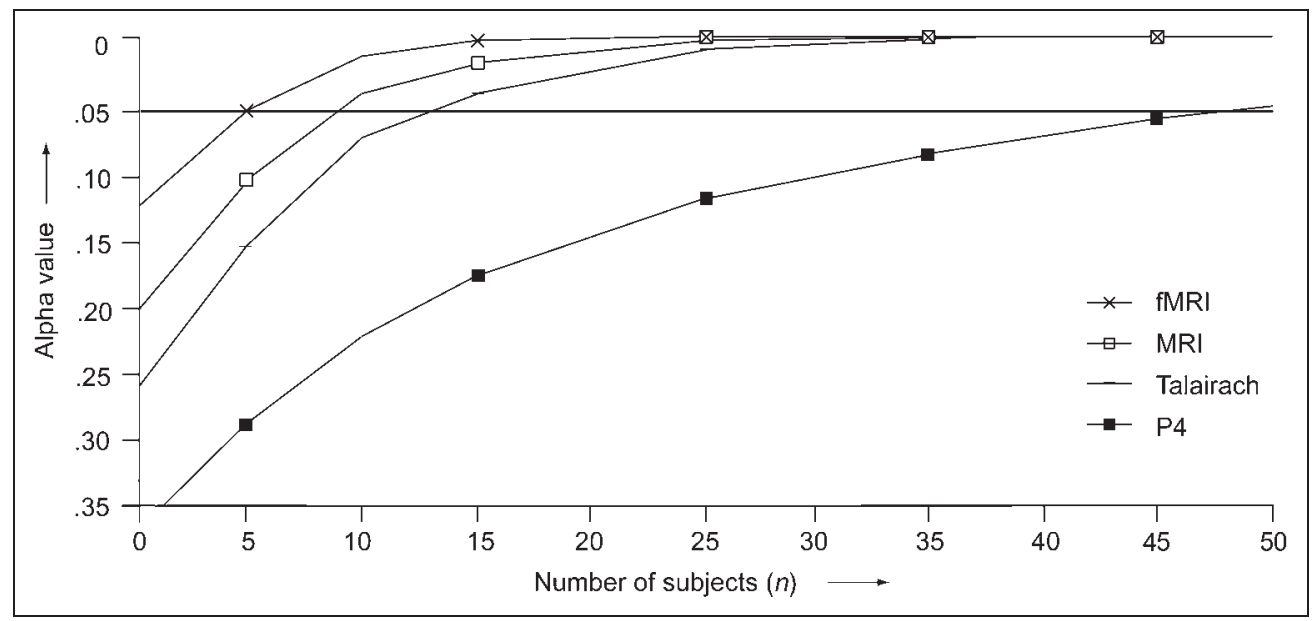




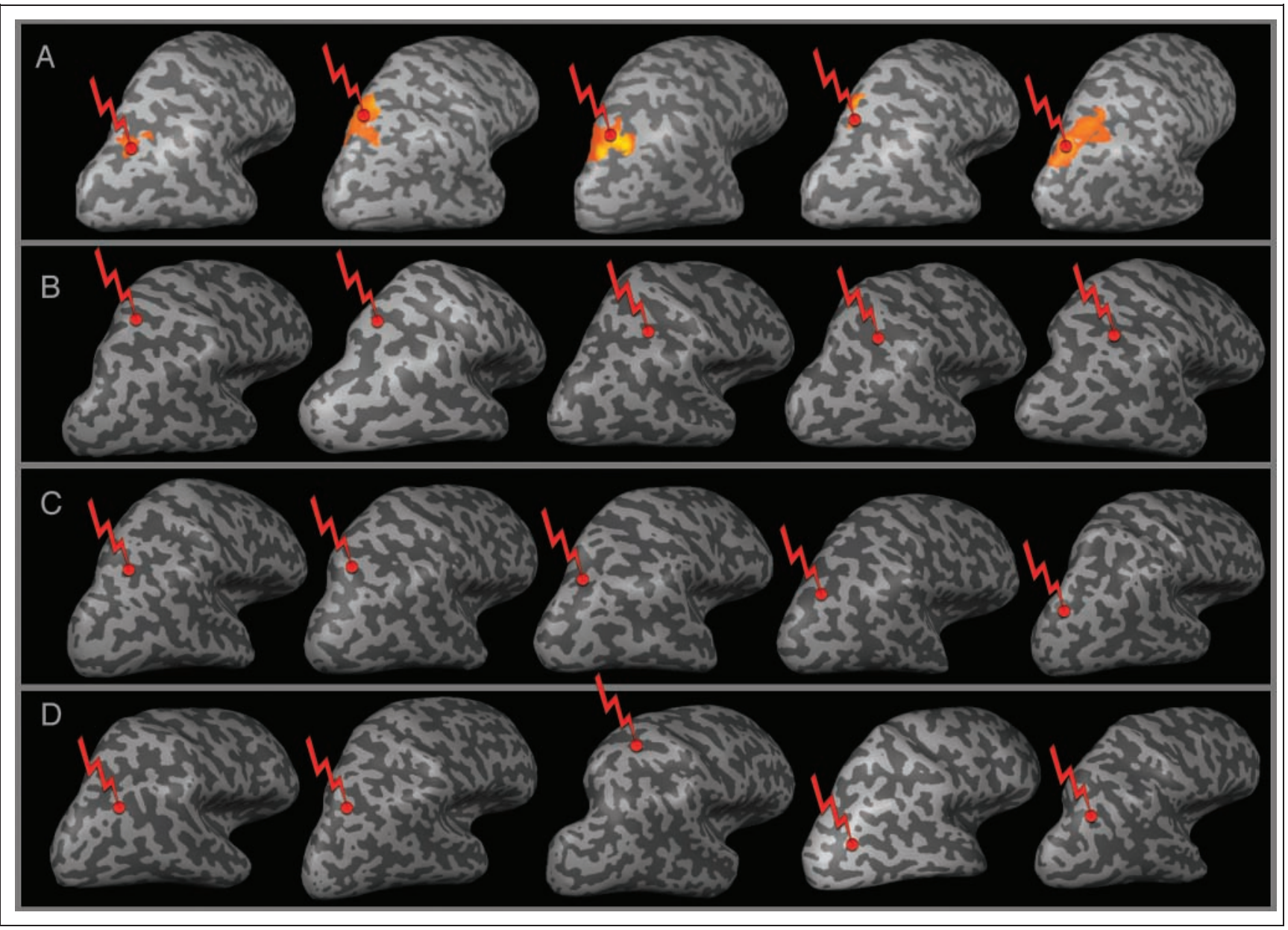

Figure 6. Segmentations of the individual participants and their TMS stimulation site. The top row shows IPS activation as a function of the SCE in the right hemisphere (A). The capital letters indicate the approaches for TMS target site determination, with (A) for fMRI-guided neuronavigation, (B) for MRI-guided neuronavigation, (C) for neuronavigation based on Talairach coordinates, and (D) for TMS based on the 10-20 EEG system. The red spheres indicate the site of the maximal stimulation.

TMS neuronavigation approach, the individual fMRI results led to a large interindividual variability of the stimulated "hot spot" defined as the strongest fMRI activity underlying the SCE ( $S D$ in $x=5.6, y=4.7, z=8.6)$. The anatomically defined right IPS target site resulted in a considerable interindividual variance in corresponding Talairach coordinates ( $S D$ in $x=10.9, y=6.2, z=2.4$ ), and even larger interindividual variances were revealed in the individual Talairach coordinates underlying the $\mathrm{P} 4$ target TMS stimulation site ( $S D$ in $x=7.0, y=19.8$, $z=19.2$ ).

In order to assess and better compare the variability of the TMS target site between the four different TMS approaches, Euclidean distances (ED) between the actual target site of stimulation and two reference target points were calculated. The first reference target point was defined as the mean Talairach coordinates of the fMRI group analysis which exhibited the strongest SCE (Site 1; Talairach coordinates $[x, y, z]=22,-68,39$ ). Although this site is likely to most closely resemble the true target area, it may bias calculations of variability. This reference target point is calculated from fMRI data acquired from subjects in the first approach, which may lead to an underestimation of variability in this approach. Therefore, EDs were additionally calculated to a reference target point based on a recent meta-analysis (Cohen Kadosh et al., 2008; Site 2; Talairach coordinates [x,y, $z]=37,-46,42)$. This enabled us to indicate in millimeters the exact distance from each actual TMS target stimulation site per subject and per approach to both of these reference points. For the fMRI-guided TMS approach, the average ED between each individual target TMS site and Site 1 was $11 \mathrm{~mm}(S D=3.5$; variance $=$ 12.2). For the MRI-guided TMS approach, the average $\mathrm{ED}$ was $24 \mathrm{~mm}(S D=7.2$; variance $=52.4)$, and for the P4 approach $25 \mathrm{~mm}(S D=11.7$, variance $=136.7)$. ED to Site 2 was $29 \mathrm{~mm}(S D=3.6$; variance $=13.1)$ for the fMRI-guided TMS approach, $13 \mathrm{~mm}(S D=8.9$; variance $=$ 79.6) for the MRI-guided TMS approach, and $36 \mathrm{~mm}(S D=$ 8.0 , variance $=63.4$ ) for the $\mathrm{P} 4$ approach (Table 3 ). This again clearly demonstrated how much the exact site of stimulation can vary between participants and between approaches. The maximal difference between the reference target point (Site 1) and the actual TMS stimulation 
Table 2. Talairach Coordinates of Target Sites for Each Participant and Euclidean Distance of the Individual Target Site to Site 1 (Talairach Coordinates $[22,-68,39]$ ) and to Site 2 (Talairach Coordinates $[37,-46,42]$ )

\begin{tabular}{lcccccc}
\hline \multirow{2}{*}{ Approach } & & & \multicolumn{5}{c}{$\begin{array}{c}\text { Distance } 1 \\
(\mathrm{~mm})\end{array}$} & $\begin{array}{c}\text { Distance } 2 \\
(\mathrm{~mm})\end{array}$ \\
\hline fMRI & P1 & 29 & -67 & 28 & 13 & 26 \\
& P2 & 16 & -70 & 47 & 13 & 32 \\
& P3 & 23 & -72 & 37 & 5 & 30 \\
& P4 & 18 & -60 & 47 & 12 & 24 \\
Anatomical & P6 & 21 & -58 & 52 & 16 & 22 \\
& P5 & 16 & -70 & 47 & 10 & 32 \\
& P7 & 20 & -59 & 51 & 15 & 23 \\
& P8 & 41 & -46 & 47 & 30 & 6 \\
Palairach & P9 & 40 & -48 & 47 & 28 & 6 \\
& P10 & 40 & -48 & 48 & 28 & 7 \\
& P11-15 & 22 & -68 & 39 & 0 & 27 \\
& P16 & 37 & -73 & 37 & 16 & 27 \\
& P17 & 32 & -82 & 28 & 17 & 39 \\
& P18 & 19 & -37 & 67 & 42 & 32 \\
& P19 & 33 & -86 & 15 & 32 & 48 \\
& P20 & 34 & -79 & 33 & 19 & 34 \\
\hline
\end{tabular}

Talairach conventions: $x=$ left to right, $y=$ back to front, $z=$ bottom to top.

site was found to be as large as $42 \mathrm{~mm}$, revealed in one participant stimulated with TMS over P4 (Table 2).

\section{DISCUSSION}

Our aim in this study was to quantify the influence of different TMS coil positioning approaches on TMSinduced behavioral effects in cognitive studies. In all four TMS positioning approaches, participants were stimulated using either real or sham TMS. In addition, a no-TMS baseline was recorded. The results and subsequent power calculations revealed the relative efficacy of the four methods compared.
Without TMS, participants showed a significant SCE as measured by a significant difference between congruent and incongruent trials for both RTs and accuracy. This significant SCE comprised a facilitatory component, revealing that participants were faster in their decision when the task-irrelevant dimension of the stimuli (physical size) was congruent with the task-relevant dimension (numerical size), as well as an interference component, revealing a decrease in performance in case of a dimension conflict. In all four approaches, sham TMS had no effect on this SCE. However, when contrasting real TMS versus sham TMS, thus calculating the net impact of right parietal TMS on the SCE, a systematic difference between the four approaches was found. For the individual fMRI-guided TMS neuronavigation approach, a significant difference between real and sham TMS in terms of a significantly reduced SCE was revealed. Moreover, there was a significant interaction between TMS and congruency. The other three approaches of TMS coil positioning showed a smaller reduction in SCE than that obtained with individual fMRI-guided TMS neuronavigation. This suggests that the four different TMS coil positioning approaches do not differ qualitatively in their TMS-induced effects but in the magnitude of their respective effect sizes, and thus, in the number of participants required to reveal the observed TMS-induced changes as being statistically significant. Accordingly, when calculating the effect size of the SCE reduction per approach, the effect size in the fMRI-guided TMS neuronavigation was largest (Cohen's $d=1.13$ ), followed by the effect size for the MRI-guided TMS neuronavigation (Cohen's $d=0.82$ ), the effect size for the TMS neuronavigation based on group Talairach coordinates (Cohen's $d=0.67$ ), and finally, smallest for the TMS over P4 approach (Cohen's $d=$ $0.34)$. A subsequent power analysis revealed that while for fMRI-guided neuronavigation five participants were sufficient to find a significant effect, the number of necessary participants increased to $n=9$ for MRI-guided neuronavigation, to $n=13$ for TMS based on group Talairach coordinates, and to $n=47$ when applying TMS over P4.

Initially, all four approaches of positioning the TMS coil attempted to denote the same area within the right IPS. Yet, the differences in the TMS-induced effect sizes show this was not the case. In order to quantify the differences in the parietal target stimulation site within and between the four approaches that might underlie these

Table 3. Averaged Euclidean Distance of the Individual Target Site to Site 1 (Talairach Coordinates [22, -68, 39]) and Site 2 (Talairach Coordinates [37, -46, 42]), Averaged per Approach for TMS Target Site Determination

\begin{tabular}{lcccccc}
\hline Approach & Distance $1(\mathrm{~mm})$ & $S D$ & Variance & Distance $2(\mathrm{~mm})$ & $S D$ & Variance \\
\hline fMRI & 11 & 3.5 & 12.2 & 29 & 3.6 & 13.1 \\
Anatomical & 24 & 7.2 & 52.4 & 13 & 8.9 & 79.6 \\
Talairach & 0 & 0 & 0 & 27 & 0 & 0 \\
P4 & 25 & 11.7 & 136.7 & 36 & 8.0 & 63.4 \\
\hline
\end{tabular}


effect size differences, we also assessed the brain coordinates of the actual parietal stimulation site in each participant using frameless stereotaxy. This calculation revealed that the actual TMS target site differed considerably between subjects and within methods. The variability between subjects and within methods was assessed by calculating the variance in ED to two reference sites: the mean group fMRI coordinates of the current subjects sample (Site 1) and standardized Talairach coordinates of a respective meta-study derived from the literature (Site 2). Although mean distances indeed differed between these two references, both sites supported the same conclusion of the variability being largest when targeting P4 and structural MRI-guided anatomical sites, followed by fMRI-guided sites.

In general, this interindividual variance within each approach is neither surprising nor necessarily problematic. Large intersubject variability has been reported along the IPS, both anatomically (Zilles, Eickhoff, \& PalomeroGallagher, 2003) and functionally (Cohen Kadosh et al., 2007). With regard to the question of an optimal TMS coil positioning approach, this quantified interindividual difference of parietal fMRI activity, in fact, demonstrates the necessity of considering this variability in order to avoid suboptimal stimulation. Indeed, neglect of this functional intersubject variability and the resulting mistargetting of magnetic stimulation may have contributed, at least partly, to the null results of previous TMS interference studies using magnitude comparison tasks (T. Gebuis, unpublished Master's thesis, Maastricht University, The Netherlands), including the size congruity paradigm (Walsh \& Pascual-Leone, 2003). Here, we accounted for this functional variability using individual fMRI-guided TMS neuronavigation, and by doing so, we indeed revealed the strongest behavioral effects compared to all other TMS coil positioning approaches. The second strongest effect sizes were revealed when using MRI-guided TMS neuronavigation. Although this approach does consider the interindividual variance in anatomical brain structure, it, unlike the fMRI-guided neuronavigation approach, neglects potential interindividual differences in structure-function relationships. Hence, although the exact same anatomical brain region along the IPS was targeted in each participant, the revealed interindividual differences in the functional "hot spot" of task-related brain activity were neglected. This might have resulted in a slightly suboptimal stimulation in some of the participants, leading to a reduced effect size of the TMSinduced behavioral changes. However, as the power analysis revealed, this approach would have resulted in a significant behavioral effect when increasing the sample size from five to nine participants.

When applying TMS based on the group Talairach coordinates, the TMS-induced behavioral effect sizes were also slightly reduced, both in comparison to the individual fMRI-guided as well as the individual MRIguided neuronavigation approaches. This probabilistic approach (Paus, 1999; Paus et al., 1997) uses frameless stereotaxy in order to navigate the TMS coil to specific standardized brain coordinates, for example, Talairach coordinates that have been reported in the literature to be functionally activated during the execution of the respective task. Although this approach considers not only structural but also functional imaging data of the respective cognitive task, it still neglects the unique individual structure-function relationship by assuming perfect consistency across individuals in the location of taskrelated activations in standardized stereotaxic space. In our study, the revealed large interindividual variance in task-related functional parietal activity changes considerably hampered the accuracy of this approach. As a consequence, the TMS-induced behavioral effect sizes during this TMS positioning approach were again smaller as compared to the individual imaging-guided results. However, as the power analysis revealed, this approach would also have resulted in a significant behavioral effect when increasing the sample size to 13 participants.

Finally, although the revealed interindividual variance of the actual TMS target stimulation site during the individual fMRI-guided and MRI-guided TMS neuronavigation approach represent the useful and important consideration of brain and task-relevant interindividual differences, the large interindividual variance of the actual parietal target region during the $\mathrm{P} 4$ stimulation approach was mainly caused by the oversimplified assumption of intersubject consistency between scalp landmarks and underlying anatomical brain regions. This anatomical landmark approach neither accounted for interindividual differences in the functional architecture of the brain (i.e., interindividual differences in structure-function relationships), nor for the existing interindividual differences in the actual correspondence between specific scalp landmarks and underlying brain anatomy (Okamoto et al., 2004; Herwig et al., 2003). Although the 10-20 EEG system considers differences in absolute head sizes between participants by measuring the relative distances between individual cranial landmarks, interindividual asymmetries and deformities of the skull and brain still cause considerable inaccuracy of electrode positioning (Towle et al., 1993; Myslobodsky \& Bar-Ziv, 1989; Habib, Renucci, Vanier, Corbaz, \& Salamon, 1984). Accordingly, Herwig et al. (2003) evaluated and quantified several electrode positions and found interindividual variations concerning the actual correspondence to underlying anatomical brain regions. Here, our cognitive TMS study indeed showed that, during this TMS coil positioning approach, the revealed TMS-induced behavioral effect sizes were the smallest as compared to the other approaches. In fact, the conducted power analysis revealed that in order to still reveal a significant behavioral effect despite the inaccurate coil positioning, 47 participants would need to be tested.

Our findings clearly document a graded difference between the four TMS coil positioning approaches with 
the individual fMRI-guided TMS neuronavigation yielding the strongest and the $\mathrm{P} 4$ stimulation approach yielding the smallest behavioral effect size. Although all four approaches account for different aspects and different levels of brain- and task-related interindividual variances, some still either underestimated (probabilistic approach) or overestimated ( $\mathrm{P} 4$ approach) the variability in the location of the SCE that is present between individuals. In this respect, our findings suggest that in case of cognitive studies or during structure-function mappings of so-called silent brain regions, the optimal method, where possible, is to use TMS on the basis of individual functional brain imaging coordinates.

However, some words of caution are required. Although individually fMRI-guided TMS is the most accurate method, our power calculations show that structural MRI guidance or mean Talairach coordinates with frameless stereotaxy are sufficient if the group size is large enough. Moreover, we investigated the functional relevance of one particular region of the IPS for one cognitive function and the extent to which our findings can be applied to other studies is uncertain. In fact, given the particularly large interindividual variability along the IPS (Zilles et al., 2003), the particular cognitive function investigated in this study may have biased our findings by specifically emphasizing the advantages of an individual fMRI-guided TMS neuronavigation as the only approach which explicitly accounts for these interindividual differences in the functional architecture of the brain. It may be that for other brain regions or other cognitive tasks, the interindividual differences in brain anatomy and structure-function relationships are less pronounced, thus reducing the difference between fMRI-guided, MRIguided, and probabilistic approaches, making these latter two approaches an acceptable compromise between stimulation accuracy and pre-experimental effort. However, one can be confident that individual fMRI-guided TMS will be the most accurate and that 10-20 system coordinates will have least power in cognitive studies.

It is also important to note that the question of determining the optimal TMS stimulation approach during cognitive studies is not sufficiently addressed by merely focusing on positioning the coil above the respective target stimulation site. In addition to the differential influence of different TMS coil positioning approaches as described above, different coil rotations above the exact same target stimulation site similarly influence the revealed behavioral effect sizes of TMS. In a recent study, Kammer, Vorwerg, and Herrnberger (2007) used TMS to stimulate the visual cortex and investigated the dependency of phosphene thresholds on TMS current orientation. Importantly, with the aim of an advanced TMS neuronavigation system which allows storing the exact position and orientation of the TMS coil relative to an individual 3-D anatomical MR scan, the authors were able to empirically relate the individual neuroanatomy in terms of site and orientation of the stimulated gyrus in the occipital cortex to the optimal current direction of TMS. They revealed that the optimal current orientation runs perpendicular to the underlying gyrus. Based on these findings, Kammer et al. (2007) conclude that similar to the motor system, the visual system behaves anisotropically when depolarized by TMS, and because this anisotropy might very well be a general feature all over the cortex, TMS coil orientation should always be considered when stimulating any cortical region. In addition, interindividual differences in scalp-to-cortex distance requires consideration of individually dosed TMS intensities as the exact same target site, and coil orientation might still result in different TMS-induced electric field strengths between participants with varying absolute distances between the TMS coil and the respective target stimulation site (Knecht, Sommer, Deppe, \& Steinstrater, 2005). Finally, in a series of studies, Wagner et al. (2008) demonstrated the need to account for the effects of the specific electric characteristics of cortical tissue. The authors evaluated the induced current density in a realistic model of the human head when being exposed to a time-varying magnetic field. The tissue electric properties were varied to ascertain their influence on the induced currents. Interestingly, current density components normal to the tissue interface were shown to exist in all solutions within the cortex, contrary to the predictions of models that rely on symmetrical geometries. Additionally, modifications in the cortical geometry were shown to perturb the field and current density distributions in magnitude, location, and orientation, such that the site of activation could be considerably altered, particularly in the case of, for example, electrical and anatomical changes following a stroke (Wagner et al., 2006) or brain atrophy (Wagner et al., 2008).

In light of these and our own findings, future software developments are still needed in order to simultaneously account for all of these influencing factors. In this respect, an idealized TMS coil positioning approach would thus be based on individual fMRI data of a given participant, using a frameless stereotaxic system which allows on-line and real-time TMS neuronavigation to a specific target stimulation site while at the same time enabling real-time determination of the optimal target TMS coil orientation, on-line dosing of the optimal TMS intensity based on individual scalp-to-target distance measurements, as well as on-line and real-time modeling of the induced current density distributions and their interaction with the specific tissue electric properties as present at the specific TMS target stimulation site.

\section{Acknowledgments}

A. T. S. and T. S. were supported by a grant from the Netherlands Organization for Scientific Research (NWO; grants 452-06-003 and 400-04-215). We thank our medical supervisor Cees van Leeuwen and our independent physician Martin van Boxtel. 
Reprint requests should be sent to Alexander T. Sack, Department of Cognitive Neuroscience, Faculty of Psychology, Maastricht University, P.O. Box 616, 6200 MD Maastricht, The Netherlands, or via e-mail: a.sack@psychology.unimaas.nl.

\section{REFERENCES}

Andoh, J., Artiges, E., Pallier, C., Riviere, D., Mangin, J. F., Cachia, A., et al. (2006). Modulation of language areas with functional MR image-guided magnetic stimulation. Neuroimage, 29, 619-627.

Aron, A. R., Robbins, T. W., \& Poldrack, R. A. (2004). Inhibition and the right inferior frontal cortex. Trends in Cognitive Sciences, 8, 170-177.

Boroojerdi, B., Foltys, H., Krings, T., Spetzger, U., Thron, A., \& Topper, R. (1999). Localization of the motor hand area using transcranial magnetic stimulation and functional magnetic resonance imaging. Clinical Neurophysiology, 110, 699-704.

Boynton, G. M., Engel, S. A., Glover, G. H., \& Heeger, D. J. (1996). Linear systems analysis of functional magnetic resonance imaging in human V1. Journal of Neuroscience, 16, 4207-4221.

Cohen, J. (1988). Statistical power analysis for the behavioral sciences (2nd ed.). Hillsdale, NJ: Erlbaum.

Cohen Kadosh, R., Cohen Kadosh, K., Schuhmann, T., Kaas, A., Goebel, R., Henik, A., et al. (2007). Virtual dyscalculia induced by parietal-lobe TMS impairs automatic magnitude processing. Current Biology, 17, 689-693.

Cohen Kadosh, R., Lammertyn, J., \& Izard, V. (2008). Are numbers special? An overview of chronometric, neuroimaging, developmental and comparative studies of magnitude representation. Progress in Neurobiology, 84, 132-147.

Critchley, H. D., Tang, J., Glaser, D., Butterworth, B., \& Dolan, R. J. (2005). Anterior cingulate activity during error and autonomic response. Neuroimage, 27, 885-895.

Di Lazzaro, V., Oliviero, A., Pilato, F., Saturno, E., Dileone, M., Mazzone, P., et al. (2004). The physiological basis of transcranial motor cortex stimulation in conscious humans. Clinical Neurophysiology, 115, 255-266.

Epstein, C. M., Verson, R., \& Zangaladze, A. (1996). Magnetic coil suppression of visual perception at an extracalcarine site. Journal of Clinical Neurophysiology, 13, 247-252.

Epstein, C. M., \& Zangaladze, A. (1996). Magnetic coil suppression of extrafoveal visual perception using disappearance targets. Journal of Clinical Neurophysiology, 13, 242-246.

Ettinger, G. J., Leventon, M. E., Grimson, W. E., Kikinis, R., Gugino, L., Cote, W., et al. (1998). Experimentation with a transcranial magnetic stimulation system for functional brain mapping. Medical Image Analysis, 2, 133-142.

Feredoes, E., Tononi, G., \& Postle, B. R. (2007). The neural bases of the short-term storage of verbal information are anatomically variable across individuals. Journal of Neuroscience, 27, 11003-11008.

Gugino, L. D., Romero, J. R., Aglio, L., Titone, D., Ramirez, M., Pascual-Leone, A., et al. (2001). Transcranial magnetic stimulation coregistered with MRI: A comparison of a guided versus blind stimulation technique and its effect on evoked compound muscle action potentials. Clinical Neurophysiology, 112, 1781-1792.

Habib, M., Renucci, R. L., Vanier, M., Corbaz, J. M., \& Salamon, G. (1984). CT assessment of right-left asymmetries in the human cerebral cortex. Journal of Computer Assisted Tomography, 8, 922-927.
Hallett, M. (2000). Transcranial magnetic stimulation and the human brain. Nature, 406, 147-150.

Henik, A., \& Tzelgov, J. (1982). Is three greater than five: The relation between physical and semantic size in comparison tasks. Memory \& Cognition, 10, 389-395.

Herwig, U., Kolbel, K., Wunderlich, A. P., Thielscher, A., von Tiesenhausen, C., Spitzer, M., et al. (2002). Spatial congruence of neuronavigated transcranial magnetic stimulation and functional neuroimaging. Clinical Neurophysiology, 113, 462-468.

Herwig, U., Satrapi, P., \& Schonfeldt-Lecuona, C. (2003). Using the international 10-20 EEG system for positioning of transcranial magnetic stimulation. Brain Topography, 16, 95-99.

Jasper, H. H. (1958). Appendix to report to Committee on Clinical Examination in EEG: The ten-twenty electrode system of the International Federation. Electroencephalography and Clinical Neurophysiology, 10, 371-375.

Kammer, T., Vorwerg, M., \& Herrnberger, B. (2007). Anisotropy in the visual cortex investigated by neuronavigated transcranial magnetic stimulation. Neuroimage, 36, 313-321.

Kaufmann, L., Koppelstaetter, F., Delazer, M., Siedentopf, C., Rhomberg, P., Golaszewski, S., et al. (2005). Neural correlates of distance and congruity effects in a numerical Stroop task: An event-related fMRI study. Neuroimage, 25, 888-898.

Knecht, S., Sommer, J., Deppe, M., \& Steinstrater, O. (2005). Scalp position and efficacy of transcranial magnetic stimulation. Clinical Neurophysiology, 116, 1988-1993.

Krings, T., Buchbinder, B. R., Butler, W. E., Chiappa, K. H., Jiang, H. J., Cosgrove, G. R., et al. (1997). Functional magnetic resonance imaging and transcranial magnetic stimulation: Complementary approaches in the evaluation of cortical motor function. Neurology, 48, 1406-1416.

Myslobodsky, M. S., \& Bar-Ziv, J. (1989). Locations of occipital EEG electrodes verified by computed tomography. Electroencephalography and Clinical Neurophysiology, 72, 362-366.

Okamoto, M., Dan, H., Sakamoto, K., Takeo, K., Shimizu, K., Kohno, S., et al. (2004). Three-dimensional probabilistic anatomical cranio-cerebral correlation via the international 10-20 system oriented for transcranial functional brain mapping. Neuroimage, 21, 99-111.

Pascual-Leone, A., Bartres-Faz, D., \& Keenan, J. P. (1999). Transcranial magnetic stimulation: Studying the brain-behaviour relationship by induction of "virtual lesions". Philosophical Transactions of the Royal Society of London, Series B, Biological Sciences, 354, 1229-1238.

Pascual-Leone, A., Walsh, V., \& Rothwell, J. (2000). Transcranial magnetic stimulation in cognitive neuroscience-Virtual lesion, chronometry, and functional connectivity. Current Opinion in Neurobiology, 10, 232-237.

Paus, T. (1999). Imaging the brain before, during, and after transcranial magnetic stimulation. Neuropsychologia, 37, 219-224.

Paus, T., Jech, R., Thompson, C. J., Comeau, R., Peters, T., \& Evans, A. C. (1997). Transcranial magnetic stimulation during positron emission tomography: A new method for studying connectivity of the human cerebral cortex. Journal of Neuroscience, 17, 3178-3184.

Penfield, W. (1958). Some mechanisms of consciousness discovered during electrical stimulation of the brain. Proceedings of the National Academy of Sciences, U.S.A., 44, 51-66. 
Picard, N., \& Strick, P. L. (2001). Imaging the premotor areas. Current Opinion in Neurobiology, 11, 663-672.

Pinel, P., Piazza, M., Le Bihan, D., \& Dehaene, S. (2004). Distributed and overlapping cerebral representations of number, size, and luminance during comparative judgments. Neuron, 41, 983-993.

Robertson, E. M., Theoret, H., \& Pascual-Leone, A. (2003). Studies in cognition: The problems solved and created by transcranial magnetic stimulation. Journal of Cognitive Neuroscience, 15, 948-960.

Rushworth, M. F., Walton, M. E., Kennerley, S. W., \& Bannerman, D. M. (2004). Action sets and decisions in the medial frontal cortex. Trends in Cognitive Sciences, 8, 410-417.

Sack, A. T. (2006). Transcranial magnetic stimulation, causal structure-function mapping and networks of functional relevance. Current Opinion in Neurobiology, 16, 593-599.

Sack, A. T., Kohler, A., Linden, D. E., Goebel, R., \& Muckli, L. (2006). The temporal characteristics of motion processing in hMT/V5+: Combining fMRI and neuronavigated TMS. Neuroimage, 29, 1326-1335.

Sparing, R., Buelte, D., Meister, I. G., Paus, T., \& Fink, G. R. (2008). Transcranial magnetic stimulation and the challenge of coil placement: A comparison of conventional and stereotaxic neuronavigational strategies. Human Brain Mapping, 29, 82-96.

Stewart, L., Battelli, L., Walsh, V., \& Cowey, A. (1999). Motion perception and perceptual learning studied by magnetic stimulation. Electroencephalography and Clinical Neurophysiology Supplement, 51, 334-350.

Talairach, J., \& Tournoux, P. (1988). Co-planar stereotaxic atlas of the buman brain. New York: Thieme.
Thiel, A., Haupt, W. F., Habedank, B., Winhuisen, L., Herholz, K., Kessler, J., et al. (2005). Neuroimaging-guided rTMS of the left inferior frontal gyrus interferes with repetition priming. Neuroimage, 25, 815-823.

Towle, V. L., Bolanos, J., Suarez, D., Tan, K., Grzeszczuk, R., Levin, D. N., et al. (1993). The spatial location of EEG electrodes: Locating the best-fitting sphere relative to cortical anatomy. Electroencephalography and Clinical Neurophysiology, 86, 1-6.

Tzelgov, J., Meyer, J., \& Henik, A. (1992). Automatic and intentional processing of numerical information. Journal of Experimental Psychology: Learning, Memory, and Cognition, 18, 166-179.

Wagner, T., Eden, U., Fregni, F., Valero-Cabre, A., Ramos-Estebanez, C., Pronio-Stelluto, V., et al. (2008). Transcranial magnetic stimulation and brain atrophy: A computer-based human brain model study. Experimental Brain Research, 186, 539-550.

Wagner, T., Fregni, F., Eden, U., Ramos-Estebanez, C., Grodzinsky, A., Zahn, M., et al. (2006). Transcranial magnetic stimulation and stroke: A computer-based human model study. Neuroimage, 30, 857-870.

Walsh, V., \& Cowey, A. (2000). Transcranial magnetic stimulation and cognitive neuroscience. Nature Reviews Neuroscience, 1, 73-79.

Walsh, V., \& Pascual-Leone, A. (2003). Transcranial magnetic stimulation: A neurochronometrics of mind. Cambridge, MA: MIT Press.

Ziemann, U. (2004). TMS induced plasticity in human cortex. Reviews of Neuroscience, 15, 253-266.

Zilles, K., Eickhoff, S., \& Palomero-Gallagher, N. (2003). The human parietal cortex: A novel approach to its architectonic mapping. Advances in Neurology, 93, 1-21. 